Eur J Clin Chem Clin Biochem

$1995 ; 33: 847-853$

(ㄷ) 1995 Walter de Gruyter \& Co. Berlin - New York

\title{
System Identification of the Low-Dose Kinetics of $p$-Aminohippuric Acid
}

\author{
By Willibald Estelberger ${ }^{1}$, Sabine Zitta ${ }^{3}$, Thomas Lang ${ }^{2}$, Frank Mayer ${ }^{1}$, Astrid Mauric ${ }^{3}$, Sabine Horn ${ }^{3}$, \\ Herwig Holzer ${ }^{3}$, Walter Petek ${ }^{2}$ and Gilbert Reibnegger \\ 1 Medizinisch-Chemisches Institut \\ 2 Chirurgische Universitätsklinik, Blocklaboratorium I \\ 3 Department für Innere Medizin, Abteilung für Nephrologie \\ Karl-Franzens-Universität Graz, Graz, Austria
}

(Received May 3/August 24, 1995)

This paper, originating from a cooperation of clinical and theoretical research groups, is dedicated to Professor Dr. E. Kaiser, the mentor of such concerted efforts, on the occasion of his 70th birthday

Summary: The renal clearance of $p$-aminohippuric acid, due to tubular secretion in addition to glomerular filtration, can only be determined by kinetic experiments. Maximal information can be gained from observed temporal marker concentration profiles by fitting dynamic mathematical models of the processes involved, such as absorption, distribution, and elimination, to the kinetic data. Thereby the values of the system constants, such as fractional elimination or fractional distribution rates, and their accuracy measures are determined by methods which are based firstly on measured time-dependent data elicited in an individual test object by perturbing inputs and secondly, on mathematical formulations of prior knowledge of the underlying physiological system. Such methods of model adaptation are called system identification.

In this context a computer-based method of system identification and error estimation for the system constants of two-compartment models matched to dynamic concentration profiles of $p$-aminohippuric acid is presented. The method is used for single-injection experiments to demonstrate that such a technique is able to correctly estimate the clearance of $p$-aminohippuric acid if sufficiently long experimental protocols are chosen, and to ascertain the sufficient length of a protocol for an individual subject. The renal clearance of $p$-aminohippuric acid is known to exhibit concentration-dependence generally, but to achieve its maximal value when low doses are applied. The present study deals with the low-dose kinetics of $p$-aminohippuric acid.

\section{Introduction}

$p$-Aminohippuric acid is a marker for the assessment of renal function by kinetic experiments (1). For reasons of clinical practicability the single-injection technique is the method of choice (2), although generally the constant-infusion technique is considered as 'gold standard'. Thus, despite the old finding that clearance values calculated from constant-infusion experiments are generally damped in magnitude due to necessarily too short experimental protocols (3) the objection still prevails that bolus experiments result in overestimation of clearance values (4). Nevertheless there is a problem of systematic clearance overestimation by single-injection methods.

Computer-based modelling and optimization methods allow system constants to be determined on the basis of non-equilibrium data. This is of special value insofar as transient system responses to external inputs principally contain more information than steady-state system outputs. The essence of such evaluation techniques consists in repeated simulations of the processes involved using varied assumptions of the system constants until the minimal deviation between simulated and measured 
concentration curves is reached. Thus, the computerbased method called system identification is an extension of regression methods to dynamic models. By further extensions of this method not only the optimal system constants but also their accuracy measures can be calculated solely from the non-equilibrium data of an individual test subject. Additionally, when using sufficiently long protocols a criterion for the correctness of the values of the system constants obtained can be deduced on the basis of appropriate models.

The above method, the general solution of the linear dynamic problem comprising both single-injection and constant-infusion experiments and a computational method for identification of the system constants have been developed previously with application to the kinetics of sinistrin as a marker of glomerular filtration (5). Although the same mathematical concept is applied here to the kinetics of $p$-aminohippuric acid administered at low doses, the appropriateness of model-linearity and the pertinent protocol- and dose-requirements were investigated separately because of the combined action of glomerular filtration and tubular secretion and, consequently, the possibly concentration-dependent characteristics in the elimination of $p$-aminohippuric acid. Thus it is to be clarified whether special technical problems and errors arising from the use of infusion pumps have to be considered. In contrast to sinistrin, which is excreted with the same rate at any concentration, the application of high dosages and infusion rates of $p$ aminohippuric acid involves the problem of getting marker levels already in the range of non-linear elimination, an effect especially to be expected in cases of reduced clearance.

In this context the present study has a threefold object. The first goal is to outline the computer-based procedure of system identification for the assessment of the maximal renal clearance of $p$-aminohippuric acid. The second aim is to show that a correct and sufficiently accurate value of this clearance estimate is obtained by this method, when certain prerequisites concerning the protocol lengths and the temporal density of the concentration measurements are met. The third object is to demonstrate that, although the system identification technique can in principle be used successfully for any kind of input strategy, as has been shown for sinistrin, proper infusion rates of $p$-aminohippuric acid are difficult to choose, whereas traditional evaluation methods of constant-infusion experiments are insufficient altogether.

\section{Test Subjects and Methods}

\section{Clinical methods}

$p$-Aminohippuric acid (Nephrotest ${ }^{\circledast}$, Biologische Arbeitsgemeinschaft, Lich, Germany) was applied as intravenous bolus and by constant infusion (B. Braun, perfusor VII). Single-injection experiments were done in 11 renal patients and in 6 normal controls. Additionally, constant infusion experiments were performed with the controls. Informed consent was obtained in all cases and the studies were approved by the local ethics committee.

\section{Single-injection technique}

\section{$\cdot$}

The fasting subjects drank $500-600 \mathrm{ml}$ of water 30 minutes before the beginning of the study and remained supine during the investigation. They were kept on a diet with $200 \mathrm{mmol}$ of sodium and $1 \mathrm{~g}$ protein per $\mathrm{kg}$ body weight the day before. After drawing $1 \mathrm{ml}$ of blood for base-value determination, a dose of $500 \mathrm{mg}$ of $p$ aminohippuric acid was injected within half a minute. Thereafter blood was withdrawn at intervals of 5 minutes during the first hour and at intervals of 15 minutes during the second hour; the intervals were subsequently increased to 30 and 60 minutes. The experiments lasted from two and a half hours in the controls to twelve hours in some of the patients.

\section{Constant-infusion technique}

The test subjects were prepared in the same way as described above. After the initial drawing of blood for base-value determinations a priming dose of $p$-aminohippuric acid of about 250 to 500 $\mathrm{mg}$ was injected within half a minute. Immediately after the injection $p$-aminohippuric acid was infused continuously at a rate of about $2 \mathrm{mg} / \mathrm{min}$. Thereafter at time-points 75,105 , and 135 minutes blood samples of $1.5 \mathrm{ml}$ each were drawn for the determination of $p$-aminohippuric acid concentration. The so-called plasma clearances were calculated as the ratios of the infusion rates divided by the averages of the 3 marker plasma levels assumed to be sufficiently close to the respective steady-state values. This procedure constitutes the traditional clearance assessment by constant-infusion experiments.

\section{Laboratory methods}

The $p$-aminohippuric acid concentrations in serum, plasma, and supernatant were measured by the method of Bratton \& Marshall (6) as modified by Smith (7). Since p-aminohippuric acid is conjugated to acetylated $p$-aminohippuric acid, the acetyl residue was removed from the $\mathrm{N}$-acetyl-p-aminohippuric acid for the determination of total $p$-aminohippuric acid by hydrolysis incubating the sample in $150 \mathrm{~g} / 1$ trichloracetic acid for one hour at $95^{\circ} \mathrm{C}$. Thereafter the amino-group could be subjected to the colorimetric reaction. No special standard is required for the measurement of conjugated $p$-aminohippuric acid, since it is converted to $p$-aminohippuric acid. The method was adapted to $\mathrm{G}-450^{\circledR}$ selective analyser (Greiner, Langenthal, Switzerland) and to a Cobas Fara ${ }^{\circledR}$ (Hoffmann-La Roche, Basel, Switzerland). The linear range goes up to $300 \mathrm{mg} / \mathrm{l}$ on both devices. Thereby the sensivitity of the method corresponding to the triple range between maximum and minimum values in a sample of 10 measurements of the blank is approximately $0.6 \mathrm{mg} / 1$. The intra-assay coefficients of variation of the method including sample preparation and hydrolysis.were determined in four different concentration ranges with the following means \pm standard deviations in $\mathrm{mg} / \mathrm{l}: 1.19 \pm 0.06,2: 21 \pm 0.06$, $11.70 \pm 0.19$, and $52.83 \pm 0.57$; the respective intra-assay coefficients of variation were: $5.22 \%, 2.76 \%, 1.64 \%$, and $1.07 \%$.

\section{Pharmacokinetic system identification}

For the formal description of the low-dose kinetics of $p$-aminohippuric acid the so-called basic model of pharmacokinetics is appropriate. This linear model for the rates of change of the marker amounts in two compartments is structurally identical to that described previously for sinistrin and can be formulated by a set of two simultaneous differential equations; with constant coefficients: 
Tab. 1 Test subjects and experimental inputs.

\begin{tabular}{|c|c|c|c|c|c|c|c|c|c|}
\hline \multirow{2}{*}{$\begin{array}{l}\text { Test } \\
\text { subject }\end{array}$} & \multirow[t]{2}{*}{ Sex } & \multirow{2}{*}{$\begin{array}{l}\text { Age } \\
\text { (a) }\end{array}$} & \multirow{2}{*}{$\begin{array}{l}\text { Height } \\
\text { (cm) }\end{array}$} & \multirow{2}{*}{$\begin{array}{l}\text { Weight } \\
(\mathrm{kg})\end{array}$} & \multirow{2}{*}{$\begin{array}{l}\text { Single- } \\
\text { injection } \\
\text { dose } \\
(\mathrm{mg})\end{array}$} & \multicolumn{2}{|c|}{ Constant infusion 1} & \multicolumn{2}{|c|}{ Constant infusion 2} \\
\hline & & & & & & $\begin{array}{l}\text { dose } \\
(\mathrm{mg})\end{array}$ & $\begin{array}{l}\text { infusion rate } \\
(\mathrm{mg} / \mathrm{min})\end{array}$ & $\begin{array}{l}\text { dose } \\
(\mathrm{mg})\end{array}$ & $\begin{array}{l}\text { infusion rate } \\
(\mathrm{mg} / \mathrm{min})\end{array}$ \\
\hline MA & a & 34 & 173 & 88 & 500 & 260 & 2.3 & 492 & 2.9 \\
\hline $\mathrm{ZK}$ & $\hat{d}$ & 30 & 176 & 77 & 500 & 347 & 2.9 & 431 & 2.6 \\
\hline $\mathrm{HV}$ & q & 44 & 163 & 63 & 500 & 274 & 1.7 & 352 & 1.4 \\
\hline $\mathrm{ZH}$ & q & 56 & 165 & 70 & 500 & 246 & 1.9 & 392 & 1.9 \\
\hline SK & $\vec{a}$ & 36 & 177 & 70 & 500 & 297 & 2.7 & 392 & 2.5 \\
\hline- & - & - & - & $i$ & - & - & - & 560 & 13.5 \\
\hline $\mathrm{BB}$ & o & 30 & 171 & 64 & 500 & 210 & 1.9 & 358 & 2.1 \\
\hline
\end{tabular}

The line with dashes indicates a third constant-infusion experiment done in test subject SK.

$$
\begin{aligned}
& \mathrm{dx}_{1} / \mathrm{dt}=\mathrm{f}(\mathrm{t})-\left(\mathrm{k}_{01}+\mathrm{k}_{21}\right) \mathrm{x}_{1}+\mathrm{k}_{12} \mathrm{x}_{2} \\
& \mathrm{dx}_{2} / \mathrm{dt}=\mathrm{k}_{21} \mathrm{x}_{1}-\mathrm{k}_{12} \mathrm{x}_{2}
\end{aligned}
$$

The input function of an experiment consisting of a bolus injection followed by constant infusion and the initial marker amounts are given by Equations 3 to 6,

$$
\begin{aligned}
& \mathrm{f}(\mathrm{t})=\mathrm{D} / \tau, \text { if } 0 \leq \mathrm{t}<\tau \\
& \mathrm{f}(\mathrm{t})=\rho, \text { if } \tau \leq \mathrm{t}<\mathrm{t}_{\mathrm{c}} \\
& \mathrm{x}_{1}(0)=\mathrm{c}_{1}(0) \mathrm{V}_{1}=\mathrm{x}_{10} \\
& \mathrm{x}_{2}(0)=\mathrm{c}_{2}(0) \mathrm{V}_{2}=\mathrm{c}_{2}(0) \mathrm{V}_{1}\left(\mathrm{k}_{21} / \mathrm{k}_{12}\right)=\mathrm{x}_{20}
\end{aligned}
$$

The fitting of the model solution to the experimental plasma concentration data measured over a sufficiently long time period can be done by a method for searching the minimum of a criterion measuring the deviation between theoretical and experimental concentration profiles:

$$
E=\Sigma\left(c_{\text {theor }}\left(t_{i}\right)-c_{\text {exp }}\left(t_{i}\right)\right)^{2},(i=1 \ldots n)
$$

The identification of the model is done by an optimization algorithm allowing one to estimate the optimally fitting values of the independent system constants $k_{01}, k_{21}, k_{12}$, and $V_{1}$ as well as of the dependent constants such as the permeability time constant $t_{21}=\ln (2) / k_{21}$ or the peripheral volume $V_{2}=V_{1}\left(k_{21} / k_{12}\right)$. The 'noise' in the experimental data can be taken into account by means of a Monte-Carlo technique for the generation of artificial protocols by superposition of random numbers on the optimal model time function (8). The random numbers are taken from a Gaussian distribution with mean zero and a standard deviation given by the following expression

$$
s=(E /(n-4))^{1 / 2}
$$

About 100 artificial protocols are created in this way and themselves subjected to the identification procedure. The resulting constellations of the system constants are evaluated statistically for the determination of the means of the system constants and their standard deviations.

\section{Results}

In 6 normal controls both single-injection and two consecutive constant-infusion experiments with low infusion rates were done. Táble 1 shows the characteristics of these test subjects together with the inputs of the three experiments. In one test subject $(\mathrm{ZH})$ one of the two constant-infusion experiments failed so that the series of concentration measurements was too unreliable for evaluation by system identification.
Figure 1 shows the estimates of the maximal $p$-aminohippuric acid clearance obtained by system identification of the single-injection and the two consecutive constant-infusion experiments as well as the clearance estimates derived for the same two constant-infusion experiments by means of the traditional assessment technique. The standard deviations of the estimates obtained by system identification are determined by the Monte Carlo method for the individual protocols.

In one of the normal test subjects (SK) a third constantinfusion experiment with a high infusion rate was done. The concentration outputs for the single-injection experiment, a constant-infusion experiment with a low infusion rate, and a constant-infusion experiment with a high infusion rate are shown in figure 2 . Therein the model-adapted and the experimental concentration profiles are illustrated. The bolus doses administered within half a minute were 500,297 , and $560 \mathrm{mg}$ in the singleinjection experiments, respectively. The respective infu-

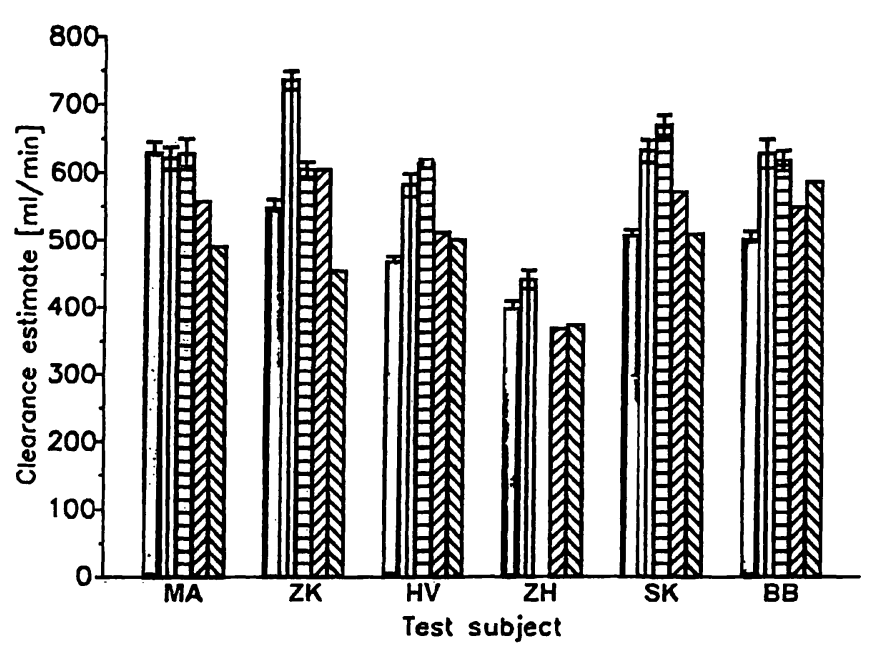

Fig. 1 Histogram of clearance estimates obtained by system identification of single-injection and constant-infusion experiments and traditional evaluation of constant-infusion experiments.

- Single injection, 血 Constant infusion 1, 目 Constant infusion 2, Constant infusion 1 (traditional), $\mathbb{Q}$ Constant infusion 2 (traditional). 
sion rates were $0,2.7$, and $13.5 \mathrm{mg} / \mathrm{min}$. The following clearance estimates were obtained: $505.8 \pm 9.3,668.9$ \pm 14.6 , and $555.3 \pm 12.9 \mathrm{ml} / \mathrm{min}$. The respective estimates of the central volumes $V_{1}$ were: $6.7 \pm 0.24,9.0$ $\pm 0.3,6.4 \pm 9.21$. Correspondingly the estimates of the peripheral volumes $\mathrm{V}_{2}$ were: $8.8 \pm 0.6,7.3 \pm 0.8,7.8$ $\pm 1.2 \mathrm{l}$. Finally, the respective estimates of the permeability time constants $t_{21}$ were: $9.1 \pm 0.7,11.4 \pm 1.3$, $12.9 \pm 1.1 \mathrm{~min}$.

Figure 3 shows the results of model-fitting to a marker profile of a single-injection experiment for a functionally anephric renal patient with an extreme extent of overhydration. This experiment lasted almost eight

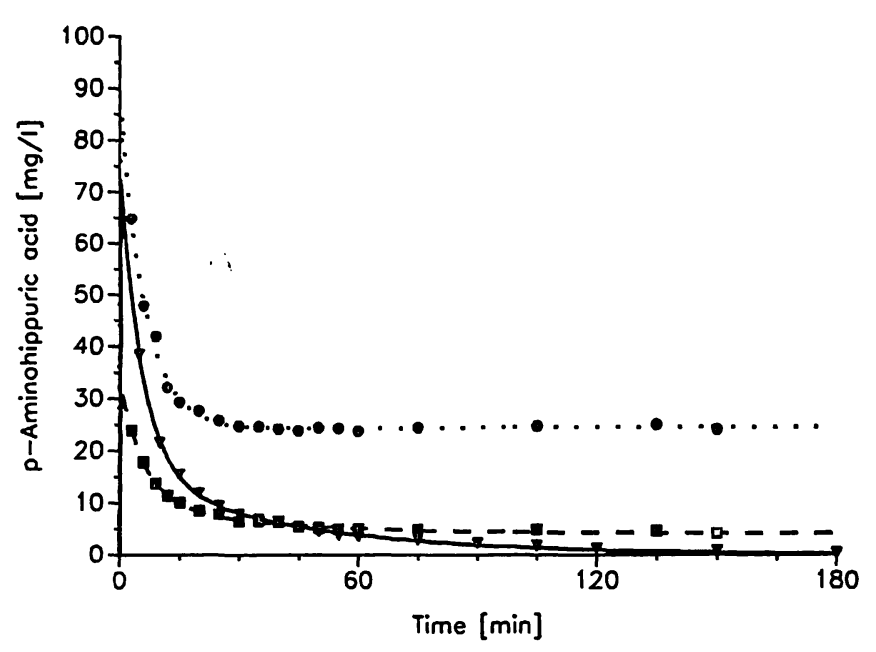

Fig. 2 Comparison of single-injection with constant-infusion experiments in a normal control (SK) using different doses and infusion rates. Measured plasma concentrations values of $p$-aminohippuric acid combined with $\mathrm{N}$-acetyl-p-aminohippuric acid together with fitted contours of single-injection experiment $(-\nabla-)$ and of constant-ifusion experiments with low (---n---) and high (...०...) infusion rates, respectively.

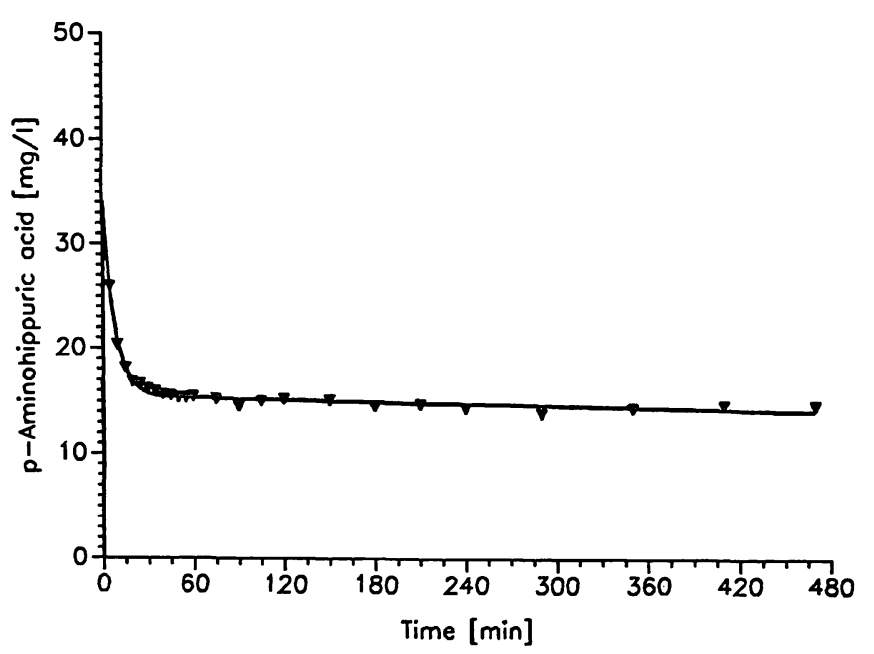

Fig. 3 Single-injection experiment in an overhydrated patient with an extreme renal insufficiency (FA). Measured plasma concentration values of $p$-aminohippuric acid combined with $\mathrm{N}$-acetyl$p$-aminohippuric acid in single-injection experiment together with fitted curve $(-\nabla-)$. hours. A dose of $500 \mathrm{mg} p$-aminohippuric acid was applied within half a minute. The system constants obtained were: clearance $=6.7 \pm 1.6 \mathrm{ml} / \mathrm{min}, \mathrm{V}_{1}=14.4$ $\pm 0.5 \mathrm{l}, \mathrm{V}_{2}=17.6 \pm 0.5 \mathrm{l}$, and $\mathrm{t}_{21}=9.6 \pm 1.0 \mathrm{~min}$.

Figure 4 illustrates that the clearance estimates derived from the single-injection experiment done in the overhydrated patient obviously depend on the experiment duration and that the calculated clearance values converge towards a limit value with increasing protocol length.

In 11 renal patients single-injection experiments were done. As table 2 shows, this group consists of 6 normally hydrated renal patients and 5 renal patients with overhydration. In table 2 the clearance estimates and their respective error measures derived from long protocols are compared with those calculated on the basis of short protocols of only 2 hours.

\section{Discussion}

As figure 1 reveals, the traditional clearance estimates are only poorly reproducible; both low and high clearance estimates occur in the repeated experiments. In addition, an inclination towards damped magnitudes is exhibited by these values. The random fluctuations are a consequence of the small sample size of only 3 data points, whereas the systematic bias is due to the necessary experimental practice of too early sampling.

The estimates gained by system identification of the two constant-infusion experiments show a systematic tendency towards values higher than those gained by the single-injection method. In principle this systematic deviation from the single-injection estimates could be due to either physiological dosage effects or to technical

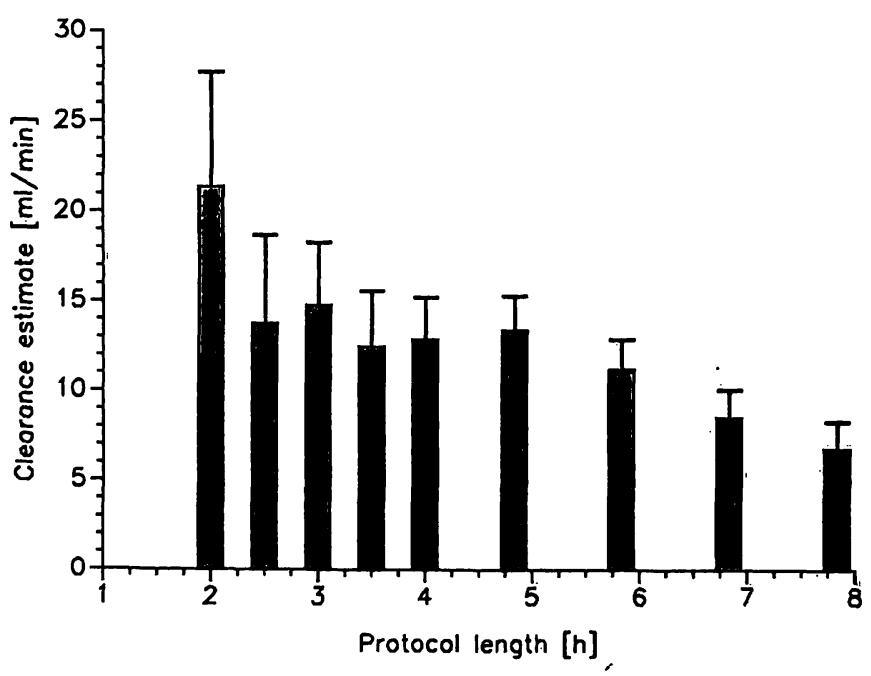

Fig. 4 Graph of protocol-length dependence of clearance estimates and standard deviations derived by Monte Carlo simulation for an overhydrated patient with an extreme renal insufficiency (FA). 
Tab. 2 Dependence of clearance estimates and variances on protocol length.

\begin{tabular}{|c|c|c|c|c|c|c|c|c|c|c|}
\hline Subject & Sex & $\begin{array}{l}\text { Age } \\
\text { (a) }\end{array}$ & $\begin{array}{l}\text { Height } \\
\text { (cm) }\end{array}$ & $\begin{array}{l}\text { Weight } \\
\text { (kg) }\end{array}$ & Diagnosis & $\begin{array}{l}t_{c} \\
(h)\end{array}$ & $\begin{array}{l}\mathrm{C}_{\text {PAH }} \\
(\mathrm{ml} / \mathrm{min})\end{array}$ & $\begin{array}{l}\mathrm{S}_{\mathrm{CPAH}} \\
(\mathrm{ml} / \mathrm{min})\end{array}$ & $\begin{array}{l}\mathrm{C}_{\mathrm{PAH}_{2}} \\
(\mathrm{ml} / \mathrm{min})\end{array}$ & $\begin{array}{l}\mathrm{S}_{\mathrm{CPAH}_{2}} \\
(\mathrm{ml} / \mathrm{min})\end{array}$ \\
\hline $\mathrm{MH}^{*}$ & $\delta$ & 64 & 172 & 82 & $\begin{array}{l}\text { Glomerulo- } \\
\text { nephritis }\end{array}$ & 4.0 & 332.8 & 10.4 & 346.3 & 7.7 \\
\hline WR* & $\delta$ & 54 & 170 & 77 & Nephrectomy & 3.0 & 304.3 & 11.1 & 310.0 & 12.0 \\
\hline $\mathrm{PA}^{*}$ & $\delta$ & 35 & 182 & 90 & $\begin{array}{l}\text { Glomerulo- } \\
\text { nephritis }\end{array}$ & 4.0 & 285.3 & 11.8 & 295.5 & 10.1 \\
\hline LA* & 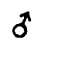 & 65 & 160 & 98 & $\begin{array}{l}\text { Glomerulo- } \\
\text { nephritis }\end{array}$ & 3.6 & 197.2 & 6.0 & 215.6 & 6.8 \\
\hline $\mathrm{PK}^{*}$ & $\sigma$ & 62 & 178 & 78 & Plasmocytoma & 4.0 & 66.2 & 4.4 & 70.9 & 10.6 \\
\hline $\mathrm{KK}^{*}$ & $q$ & 69 & 156 & 58 & $\begin{array}{l}\text { Interstitial } \\
\text { nephritis }\end{array}$ & 4.0 & 59.1 & 1.8 & 66.5 & 1.6 \\
\hline$Z^{* *}$ & $\hat{\sigma}$ & 28 & 167 & 78 & Nephrectomy & 7.0 & 174.1 & 7.1 & 194.0 & 6.5 \\
\hline TA** & q & 70 & 159 & 64 & $\begin{array}{l}\text { Morbus } \\
\text { Wegener }\end{array}$ & 4.0 & 105.5 & 4.1 & 122.0 & 4.7 \\
\hline SK** & $\delta$ & 21 & 150 & 48 & $\begin{array}{l}\text { Nephrectomy, } \\
\text { cystinosis }\end{array}$ & 13.5 & 46.9 & 1.9 & 54.4 & 2.6 \\
\hline RJ** & $\delta$ & 65 & 170 & 83 & $\begin{array}{l}\text { Diabetes } \\
\text { mellitus }\end{array}$ & 24.5 & 29.9 & 3.1 & 71.0 & 10.2 \\
\hline $\mathrm{FA}^{* *}$ & $\sigma$ & 54 & 175 & 70 & $\begin{array}{l}\text { Diabetes } \\
\text { mellitus }\end{array}$ & 7.8 & 6.7 & 1.6 & 21.5 & 6.4 \\
\hline
\end{tabular}

* renal patients without overhydration,

** overhydrated renal patients.

$t_{c}$ : length of protocol;

$\mathrm{C}_{\mathrm{PAH}}$ : clearance estimate based on full protocol,

problems associated with the infusion regimes. A dosage effect, however, seems rather unlikely, since the doses are very different, whereas the clearance values are the same in both of the two infusion experiments with just one exception. Furthermore, although there are large differences in the priming doses, the mean concentration levels reached are in the same ranges as those for the single-injection experiments as is exemplified in figure 2 except for the experiment with the very high infusion rate.

Thus, the higher clearance values of 5 of the constantinfusion experiments evaluated by system identification obviously have the trivial technical cause that the actual infusion rates were lower than the nominal ones in the 5 of the 6 cases where the discrepancies between the single-injection and the constant-infusion estimates become apparent. Evidently, the infusion rates were chosen too cautiously in order to avoid non-linear physiological elimination effects, which could have expressed themselves at least in renally insufficient test subjects. No such problems were observed previously with sinistrin, where the selected infusion rates were more than tenfold higher, since glomerular filtration is known to be independent of the plasma concentrations of the marker.

The findings in connection with figure 1 are confirmed especially by those illustrated in figure 2 . Thus, contrary to traditional expectations, the clearance estimates ob-
$\mathrm{s}_{\mathrm{CPAH}}$ : standard deviation of $\mathrm{C}_{\mathrm{PAH}}$,

$\mathrm{C}_{\mathrm{PAH}_{2}}$ : clearance estimate based on shortened protocol of 2 hours, and

$\mathrm{S}_{\mathrm{CPAH}_{2}}:$ standard deviation of $\mathrm{C}_{\mathrm{PAH}_{2}}$.

tained by constant-infusion techniques are significantly higher than that of the single-injection technique. This, however, cannot be interpreted as an indication of a nonlinear effect for the following three reasons.

Firstly, the doses and the infusion rates are chosen such that only the linear, i. e. concentration-independent physiological elimination kinetics are involved.

Secondly, the concentration-ranges of the single-injection and the constant-infusion experiments with the low infusion rates are nearly always the same. But it is these two experiments which exhibit the largest difference in their clearance estimates, whereas the clearance estimate derived from the experiment in which a high infusion rate was employed approaches that of the single-injection experiment.

Thirdly, although the marker levels of the constant-infusion experiment with the high infusion rate are higher than those of the single-injection experiment, the clearance estimate is also higher than that of the single-injection experiment. It should, however, be lower than that of the single-injection experiment, if physiological saturation effects were involved. Thus, this finding together with those of figure 1 is an indication of hidden technical problems involved in the use of infusion-pumps, a source of error arising even when steady states can be achieved. 
Despite all this evidence against both traditional and modern constant-infusion methods, the question of possible clearance overestimation by single-injection clearance assessment is to be considered. This is demonstrated in figures 3 and 4 and in table 2.

As can be seen from figure 3, there are systematic residue fluctuations of the experimental data points around the model-calculated concentration curve. This phenomenon, which has also been observed for sinistrin in cases of enlarged extracellular spaces, has consequences for the required experimental protocol lengths. Thus, if the chosen protocol-lengths are too short, there will be clearance overestimation.

Figure 4 shows that a limit value within the error boundaries obtained by the Monte Carlo method described will be reached only with a long protocol. This finding shows the necessity of convergence-testing.

In table 2 the convergence behaviour of the clearance estimates with increasing protocol lengths is studied. As the clearance comparisons of table 2 indicate, there is a protocol-length dependency of the clearance estimates of $p$-aminohippuric acid. Both the clearance estimates and their errors generally decrease with increasing protocol lengths. The required protocol length is associated with the hydration status of the test subject in any case in patients with low clearances. Thereby the hydration status can be judged from the ratio of the size of the total distribution sinistrin space derived from kinetic sinistrin experiments and an average percentage of the body weight (5).

\section{Conclusion}

System identification is a modern technique which has found entrance into areas of physiology and pathophysiology, such as in the investigation of metabolism by tracers $(9-11)$ and is to be regarded as the most exact and informative evaluation method for kinetic organ function testing. This study has shown that methods of system identification can be transferred to problems of renal function testing. Thereby the aim is to determine kinetic system constants and their accuracy measures from non-equilibrium concentration data on the basis of temporal marker profile measurements. It is self-evident that only mathematically founded model-based techniques and not any traditional rules-of-thumb, however widely accepted, are the adequate instruments for determining the correct estimates and the error ranges for the distribution volumes and the clearance from a particular experimental protocol.

By comparisons of single-injection and constant-infusion experiments in which low doses of $p$-aminohippuric acid were applied, evaluated by means of system identifcation, we found that constant-infusion techniques are inflicted with additional errors ensuing from the use of infusion-pumps. For single-injection techniques it has been shown that clearance-estimates converging to their final values can be derived from non-equilibrium concentration data, if sufficiently lon' experimental time horizons are chosen. The associated protocol-length requirements depend on the hydration status of the test subject as shown previously for sinistrin. Only system identification of non-equilibrium experiments, however, can quantitatively ascertain the sufficiency of a particular experimental protocol length.

The presented computer-based system identification method combined with a test of convergence of the clearance estimates constitutes a new approach to renal function testing $\left.{ }^{1}\right)$. It circumvents both urine collecting and infusion-pumps which both introduce additional sources of errors. It contains a self-examination for correctness of the clearance estimates obtained by testing the convergence of these values. As demonstrated there are experimental requirements concerning both the length of the experimental protocol and the temporal density of the data points. These should be chosen roughly at time-points $5,10,15,30,45,60,90,120$, 180,240 minutes from the beginning, whereby the required duration of the experiment depends on the hydration status and the expected renal efficiency.

Single-injection experiments have hitherto been evaluated by means of model-independent and curve-stripping methods (12). The modelling approach explicated in this paper goes far beyond the peeling methods of empirical exponential functions as given in the literature for bolus experiments. By mechanistic kinetic modelling the concentrations both in the central and the peripheral compartments can be taken into account as initial conditions and calculated for consideration in the clearance assessment of an immediately following experiment. Double experiments of this sort are of interest for investigation of renal functional reserve capacity as provocable e. g. by drug or diet influences. As preliminary data in moderate hypertensive patients indicate, such examinations could be of special interest in cases not yet showing any other organic manifestation of hypertension except for a reduced renal reserve capacity.

Model-oriented computer-based identification of kinetic processes also offers the chance of determining indices characterizing the saturable elimination of tubularly

\footnotetext{
1) A.computer programme for a PC running under DOS can be obtained free of charge from the author named in the address of correspondence by sending a formated 3.5" HD diskette.
} 
secreted substances when applied at high dosages (13, 14). System identification of a non-linear model of the saturable elimination process in combination with a linear model of renal excretion of sinistrin for the determination of the glomerular filtration rate should enable one to quantify the concentration-dependence of the clearance of $p$-aminohippuric acid on the basis of temporal plasma concentration data and thus to get a quantitative index of the number of active nephrons besides the measure of renal perfusion (15). An even more advanced

\section{References}

1. Weiner IM. Organic acids and bases and uric acid. In: Seldin DW, Giebisch G, editors. The kidney: physiology and pathophysiology. New York: Raven Press, 1985:1703-24.

2. Donath $\mathrm{A}$. The simultaneous determination in children of glomerular filtration rate and effective renal plasma flow by the single injection clearance technique. Acta Paediat Scand 1971: 60:512-20.

3. Berger EY, Farber SJ, Earle DP, Jackenthal R. Comparison of the constant infusion and urine collection techniques for the measurement of renal function. J Clin Invest 1948; 27:710-6.

4. Levey AS, Madaio MP, Peronne RD. Laboratory assessment of renal disease: clearance, urinalysis, and renal biopsy. In: Brenner BM, Rector FC Jr, editors. The kidney. Philadelphia: Saunders, 1991:919-68.

5. Estelberger W, Petek W, Zitta S, Mauric A, Horn S, Holzer H, Pogglitsch $\mathrm{H}$. Determination of the glomerular filtration rate by identification of sinistrin kinetics. Eur J Clin Chem Clin Biochem 1995; 33:201-9.

6. Bratton AC, Marshall EK. A new coupling component for sulfanilamide determination. J Biol Chem 1939; 128:537.

7. Smith WH, Funkelstein N, Aliminosa L, Crawford B, Graber $M$. The renal clearances of substituted hippuric acid derivatives and other aromatic acids in dog and man. J Clin Invest 1945; 24:388-404.

8. Metzler CM. Statistical properties of estimates of kinetic parameters. In: Bozler G, van Rossum JM, editors. Pharmacokinetics during drug development: data analysis and evaluation techniques. Stuttgart: Fischer, 1982:128-43.

9. Carson ER, Cobelli C, Finkelstein L. The mathematical modeling of metabolic and endocrine systems. New York: Wiley, 1982:23-35.

10. Phair RD. The role of kinetic modeling in physiology. In: Berman M, Grundy SM, Howard BV, editors. Lipoprotein kinetics and modeling. New York: Academic Press, 1982:37-40. possibility opened by modern system identification should be the quantitative investigation of the mutual inhibition of tubularly secreted substances through competition for the commonly used transport carriers (16, 17).

\section{Acknowledgements}

We thank K. Psonder and A. Meinitzer for their excellent technical assistance.

11. Carson ER, Godfrey KR, Reeve J. A review of modelling and the role of dynamic tracer studies in metabolism. In: Cramp DG, editor. Quantitative approaches to metabolism. Chichester: Wiley, 1982:1-72.

12. Valkó $\mathrm{P}$, Vajda $\mathrm{S}$. Advanced scientific computing in BASIC with applications in chemistry, biology and pharmacology. Amsterdam: Elsevier, 1989:313-7.

13. Van Ginneken CAM, Russel FGM. Saturable pharmacokinetics in the renal excretion of drugs. Clin Pharmacokinet 1989; $16: 38-54$

14. Ludden TM. Nonlinear pharmacokinetics. Clin Pharmacokinet 1991; 20 (6):429-46.

15. Estelberger W, Petek W, Pogglitsch H. Model-based determination of renal clearance from temporal venous plasma profiles of markers. In: Trappl R, editor. Cybern Syst Res; 1992 Apr 21-24; Vienna. Singapore: World Scientific, 1992; 2:893900.

16. Estelberger W, Petek W, Pogglitsch H. Simulation der sättigbaren und hemmbaren Kinetik renal-tubulär eliminierter Pharmaka. In: Pessenhofer H, editor. Biomed Tech; 1992 Sep 1719; Graz. Berlin: Schiele \& Schön, 1992:83-5.

17. Estelberger W, Weiss G, Petek W, Paletta B, Wachter H, Reibnegger G. Determination of renal clearance of neopterin by a pharmacokinetic approach. FEBS Lett 1993; 329:13-6.

Dr. Willibald Estelberger

Medizinisch-Chemisches Institut und

Pregl Laboratorium

Karl-Franzens-Universität Graz

Harrachgasse 21/2

A-8010 Graz

Austria 
